\title{
Sharing valuable image assets safely and efficiently
}

\begin{abstract}
Janice Mayo
President of OnRequest Images, brings more than 20 years of expertise in management, branding, corporate marketing and creative services to the Seattle-based company. Prior to joining OnRequest, Ms. Mayo headed marketing and creative services for Vertis where, in fewer than five years, she built a brand that was named one of "The Most Admired Companies in America" by Fortune Magazine. Formerly president of Foote, Cone and Belding Direct, and owner of her own advertising agency, Ms. Mayo has developed and managed marketing and advertising strategies for blue-chip clients nationally and internationally. A recognized expert in direct marketing, Ms. Mayo is a sought-after speaker.
\end{abstract}

Keywords: OnRequest Images, OnBrand, custom imagery, Essilor, image library, DAM

Abstract Always looking to set their brands apart from competitors, many of today's marketers are pursuing the benefits of OnBrand ${ }^{\mathrm{TM}}$ custom imagery. To maximise the use of brand-aligned images, it is necessary for marketers to store these in a secure and easily accessible location. This paper will address how marketers can effectively share, strengthen and cultivate their corporations' imagery when working with a trusted custom imagery provider that utilises DAM systems. Furthermore, this piece will cover the ins and outs of digital file sharing for custom imagery campaigns and include a real-world example of one company that managed its OnBrand imagery through a digital platform.

Journal of Digital Asset Management (2007) 3, 181-184. doi:10.1057/palgrave.dam.3650083

\section{INTRODUCTION}

In today's marketing environment, leading brands are focused on capturing the attention of consumers worldwide. Whether these brands are competing via direct marketing, outdoor advertising, online or through a handful of other media, one thing is certain: the proliferation of marketing channels has created a growing need for both campaigns and their corresponding imagery to be strategically targeted to a specific audience. As such, marketers for companies large and small have begun choosing customised, brand-aligned imagery while developing top-line marketing campaigns, in lieu of traditional stock photography options. As customised imagery increasingly comes into play, there is a greater need for the collaborative efforts of marketing, legal, creative and agency partners around the globe, which poses a new marketing challenge: effectively storing and managing proprietary photos online, in a library that can be accessed by company players at any time. In order to overcome this sizeable challenge, many marketers have come to rely on DAM tools to streamline and simplify their custom imagery workflow.
While these tools are effective at streamlining communication, it is only one phase in the process used to develop targeted campaigns. Seattle-based OnRequest Images, a leading provider of custom imagery solutions, realised the diluting effect stock photography has over brand equity and launched a service creating and managing OnBrand ${ }^{\mathrm{TM}}$ imagery for marketing campaigns. Historically, marketing teams looking for campaign imagery in a crunch have used stock photos to package around their final campaign project. While stock photography has typically been perceived as easily accessible and low-budget, it carries the heightened risk of selecting the same imagery that other corporations are using or diluting brand identity because imagery is not in sync with brand messaging. Implementing customised imagery allows marketers to capture established brand attributes through photography, therefore creating a visual connection between the brand and its message.

Many of today's leading brands have experienced the effectiveness of using customised images, while technology has further enabled the 
creation, production and internal file sharing of custom imagery to become a reality for marketing teams across the globe. There are, however, still essential issues with file sharing that must be monitored and taken into account. For example, online security issues can threaten corporate confidentiality and even a company's overall marketing strategy if breached. Add to this the increasing popularity of shared files among large marketing teams, as well as the necessity for tracking access to files companywide, and we see the critical need for a secure and highly adequate file sharing system.

\section{THE NEED FOR FILE SHARING}

For corporate marketers looking to stay ahead of competing brands, they must have the ability to track the reach and results of multiple marketing programs. To accomplish the aforementioned, improve companywide collaboration and reduce marketing costs, many companies are resorting to files on-demand to centralise their customised image libraries. Such file sharing provides the following benefits:

1. Streamlined brand consistency: Managing multiple campaigns across several departments and global office locations can be difficult. When companies employ different campaigns that are not aligned with one brand voice, it can negatively affect a brand's equity and marketing ROI. Over time, consumers begin to know a brand for its unique look and feel, allowing them to instantly recognise the brand and focus more on the advertising message. When a company is simultaneously running marketing campaigns across the globe, it is necessary to have one central image library in place so marketers are maintaining a consistent brand aesthetic while sharing peer-to-peer files.

2. Simplified global marketing: International companies market to a variety of cultural audiences each day; reaching diverse consumer segments with appropriate multicultural images can be challenging. Factors such as cultural biases, varying forms of humor and the models photographed for each campaign must be planned for and considered at all times. For example, if a US-based chain restaurant decides to run a campaign targeting Hispanic consumers in both the United States and Mexico, marketers must be able to easily access culturally accurate images of Hispanics that are customised solely to the brand. The campaign in Mexico must depict Hispanics in a Mexican setting, while the US campaign must depict Hispanics in an American setting and capture HispanicAmerican values, all while keeping imagery in one brand voice.

3. Improved collaboration: When marketers do not have access to a collection of pre-approved, customised brand images, they are often forced to obtain generic photos elsewhere. Aside from the previously mentioned brand dilution that stock photos bring about, stock imagery sites do not provide a report on which companies have purchased which images; consequently, many brands - often close competitors unknowingly use identical images in their marketing campaigns, causing an even greater decrease in brand equity and blurring of brand lines in the mind of the consumer. Online custom image libraries deplete the risk of duplicate imagery use while managing file access across the globe. Additionally, by keeping photography in one central location, brand managers can control and improve marketing collaboration companywide.

\section{SAFEGUARDING YOUR CORPORATION}

Although file sharing can greatly streamline marketing communications, making such information available companywide presents inevitable risks. Unprotected file sharing not only threatens corporate confidentiality but it can also open the door to digital asset piracy. Hackers may have the ability to view, alter, delete or steal customised image files, placing intellectual property in the hands of the public. Images that are stolen and reused deface the marketing plan and weaken brand equity when attached to another company's campaign.

Aside from security issues, companies with inefficient DAM programs become liable for their employees' actions. Without any access to a centralised image library, employees in various 
locations across the United States may purchase images with no regard to or knowledge of copyright infringement, royalty fees or permission rights. For example, few people are aware that many US landmarks cannot be included as backdrops in corporate imagery unless the user acquires the appropriate consent.

\section{SOLUTIONS FOR INADEQUATE FILE SHARING}

For corporations in need of establishing a custom image library that is highly efficient in all aspects of DAM, a few simple steps must be taken:

1. Consult an expert: Every corporation is different; the best way to resolve file sharing problems is to consult an expert who can assess your unique situation. For example, OnRequest Images has specialists who analyse corporations' individual systems while keeping in mind established brand attributes. This helps both the brand and the expert design a customised image management solution to identify and resolve brand flaws in DAM.

2. Install a secure platform: Although file sharing is a legal technology, many outside users download unprotected, copyrighted materials without gaining the proper permission. For full protection of valuable, customised digital assets, and for full control and sharing of these and other digital communication pieces, assets must be managed and maintained across multiple secure sites to protect from disaster and ensure redundancy.

3. Designate a "brand guardian": In order to guarantee images are "OnBrand," smaller corporations without brand managers should designate a "brand guardian" in the company. This individual should be in charge of approving every image used in marketing efforts companywide. By ensuring all images are inspected and protected before going public, each piece of marketing collateral used will match the desired brand aesthetic. In the long run, this will work to strengthen brand equity, marketing ROI and eventually, the company's bottom line.
To put this information in context, let us examine a brand that effectively identified and solved its need for custom imagery and management of its newly digital assets by working with OnRequest Images.

\section{ESSILOR}

The world leader in ophthalmic lenses, Essilor was aggressively looking for a solution to problems with its image database, which surfaced due to inadequate file sharing and management. Essilor turned to OnRequest Images for a Web-based solution that would house all corporate images while enabling marketers to efficiently search, track, share and manage licenses for their visual assets. While Essilor possessed thousands of photographs it had created or purchased from a number of sources, Essilor corporate marketing had no central repository for these images. As a result, individual employees were unable to track which images their co-workers had purchased, resulting in the unintentional use of duplicate images for various marketing campaigns across the company.

OnRequest Images advised Essilor to employ a photography asset management platform that would deliver the brand-building power of custom image creation and the efficiency of worldwide image sharing in one seamlessly integrated solution. This solution allowed Essilor to efficiently manage every aspect of its custom photography and share digital images across the entire organisation. Furthermore, OnRequest Images edited and key-worded approximately 1,500 of Essilor's photos, allowing company employees to effectively search every piece of imagery instantaneously.

The asset management platform also effectively solved Essilor's need for a system that would provide access to consistent, approved imagery across its various international locations. With the simple, centralised image repository and powerful search, selection and collaboration tools, Essilor employees and advertising partners around the globe could quickly choose the right imagery for advertising, promotions, marketing collateral, employee development and product launches. Any approved user with Internet capabilities had full access to search, download and use brand-approved images.

Based on the success of this platform, Essilor again turned to OnRequest Images for assistance 
with one of its premier brands, DEFINITY ${ }^{\circledR}$. In the midst of creating new collateral for an upcoming event to help drive awareness and sales for the recently re-branded campaign "WOW: Wide Open World," the company required updated and customised OnBrand imagery immediately. Essilor understood OnRequest Images could not only complete this project on time, but could also handle all creative aspects and brand elements, in addition to the complex logistics of a five-location shoot and a wide diversity of models. OnRequest Images took the project one step further, streamlining the customised image collaboration process with a proprietary automated photography production platform.

The first step was to develop an OnBrand Photography Guide ${ }^{\mathrm{TM}}$, where brand imagery consultants worked with Essilor to understand the unique personality and voice of DEFINITY, ultimately delivering a step-by-step guide detailing the most important aspects of achieving OnBrand photography. This guide was referenced during the shoot and served as the "brand imagery guide" for all future brand endeavors. OnRequest Images then targeted five locations around the globe, leveraging the resources, connections and automated workflow of its expansive team, which enabled access to locations that would otherwise be inaccessible or prohibitively expensive to book. Once locations were secured, OnRequest Images utilised its proprietary model review and selection tool to enable Essilor to review and select talent digitally in just a few hours from the comfort of its main offices. With locations and models secured, the OnRequest Images team developed a shoot that provided Essilor with even more images choices than it previously requested.

After just four days of production and more than 8,000 images shot, OnRequest Images delivered OnBrand photography that perfectly captured the new DEFINITY brand; these customised photos were quickly placed into 10 new pieces of collateral, throughout corporate presentations and onto Essilor's Web site. The combined ability of further building its custom image library while having immediate access, control and licensing over these images provided Essilor with a strategic way to intelligently strengthen its brand while increasing equity in the long run.

With the proliferation of brands competing for consumer attention around the globe, the need for companies to stand apart with one, unique brand voice is more critical than ever. Corporate marketing teams must not only realise the importance of customised, OnBrand imagery, but also take the steps necessary for creating secure and efficient images that can be shared across international borders. Customised imagery allows brands to visually connect with its audience using photography that speaks to the target consumer's lifestyle, while capturing established brand attributes through photography. Meanwhile, online file sharing systems allow marketing groups worldwide to design campaigns that are aligned with the brand and consistent with corporate messaging. By ensuring these images are available through a secure file sharing system, marketers can more efficiently and costeffectively control the invaluable identity of their brand. 